\title{
Adsorption-controlled growth of EuO by molecular-beam epitaxy
}

R. W. Ulbricht, A. Schmehl, T. Heeg, J. Schubert, and D. G. Schlom

Citation: Appl. Phys. Lett. 93, 102105 (2008);

View online: https://doi.org/10.1063/1.2973180

View Table of Contents: http://aip.scitation.org/toc/apl/93/10

Published by the American Institute of Physics

\section{Articles you may be interested in}

Epitaxial EuO thin films on GaAs

Applied Physics Letters 97, 112509 (2010); 10.1063/1.3490649

Epitaxial growth and magnetic properties of EuO on (001) Si by molecular-beam epitaxy

Applied Physics Letters 83, 975 (2003); 10.1063/1.1593832

Effect of film thickness and biaxial strain on the curie temperature of EuO

Applied Physics Letters 102, 062404 (2013); 10.1063/1.4789972

La-doped EuO: A rare earth ferromagnetic semiconductor with the highest Curie temperature

Applied Physics Letters 96, 232503 (2010); 10.1063/1.3416911

Adsorption-controlled growth of $\mathrm{BiMnO}_{3}$ films by molecular-beam epitaxy

Applied Physics Letters 96, 262905 (2010); 10.1063/1.3457786

Molecular beam epitaxy of $\mathrm{SrTiO}_{3}$ with a growth window

Applied Physics Letters 95, 032906 (2009); 10.1063/1.3184767

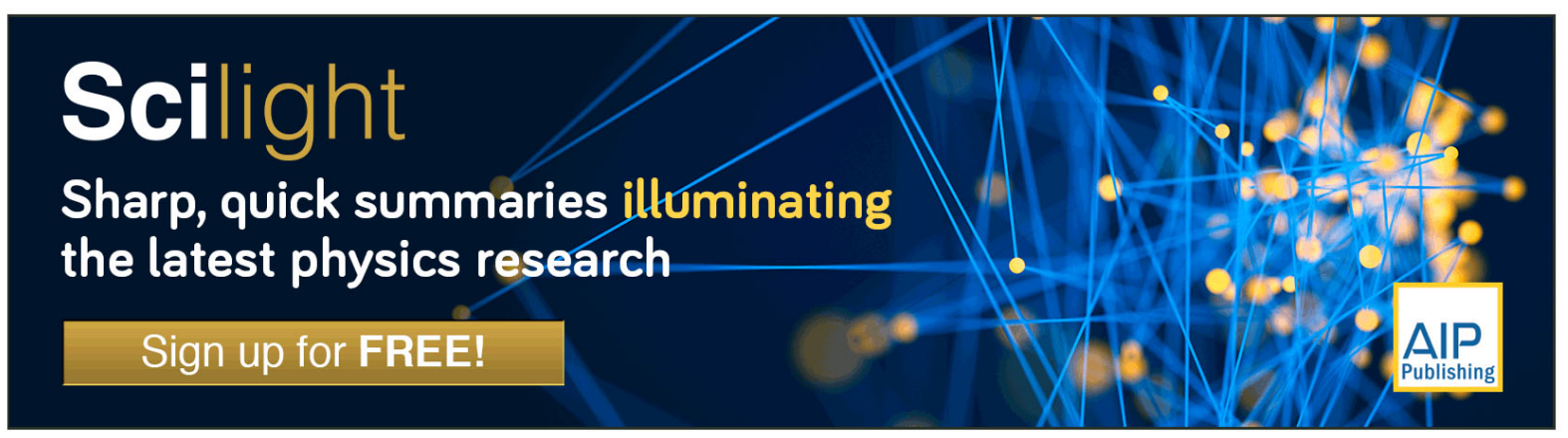




\title{
Adsorption-controlled growth of EuO by molecular-beam epitaxy
}

\author{
R. W. Ulbricht, ${ }^{1, a)}$ A. Schmehl, ${ }^{2}$ T. Heeg, ${ }^{1}$ J. Schubert, ${ }^{3}$ and D. G. Schlom ${ }^{1, b)}$ \\ ${ }^{1}$ Department of Materials Science and Engineering, Cornell University, Ithaca, New York 14853-1501, USA \\ ${ }^{2}$ Experimentalphysik VI, Elektronische Korrelationen und Magnetismus, Institut für Physik, Universität \\ Augsburg, Augsburg D-86135, Germany \\ ${ }^{3}$ Institute of Bio- and Nano-Systems (IBN1-IT), Research Centre Jülich, Jülich D-52425, Germany
}

(Received 15 February 2008; accepted 18 July 2008; published online 10 September 2008)

\begin{abstract}
Using molecular-beam epitaxy, we demonstrate the adsorption-controlled growth of epitaxial EuO films on single crystalline (110) $\mathrm{YAlO}_{3}$ substrates. Four-circle x-ray diffraction (XRD) reveals phase-pure, epitaxial, (001)-oriented films with rocking curve full width at half maxima as narrow as $34 \operatorname{arcsec}\left(0.0097^{\circ}\right)$. The critical thickness for the onset of relaxation of (001) $\mathrm{EuO}$ on (110) $\mathrm{YAlO}_{3}(\sim 2 \%$ lattice mismatch) was determined from XRD measurements to be $382 \pm 25 \AA$. A saturation magnetization of $6.96 \pm 0.07 \mu_{B} / \mathrm{Eu}$, a value close to the theoretical limit of $7 \mu_{B} / \mathrm{Eu}$, is observed. () 2008 American Institute of Physics. [DOI: 10.1063/1.2973180]
\end{abstract}

With a spin polarization exceeding $90 \%$ and its ability to be epitaxially integrated with mainstream long spin lifetime semiconductors such as silicon and $\mathrm{GaN},{ }^{1}$ the ferromagnetic semiconductor $\mathrm{EuO}$ is an attractive material for semiconductor-based spintronic devices. In the ferromagnetic phase $\left(T_{C}=69 \mathrm{~K}\right)$, the $4 f^{7}$ electron configuration of $\mathrm{Eu}^{2+}$ induces a magnetic moment of $7 \mu_{B}$ per europium atom. $^{2,3}$ Additionally, it has outstanding magneto-optical properties, with a Faraday rotation of $8.5 \times 10^{5} \mathrm{deg} / \mathrm{cm}$ in a field of $2 \mathrm{~T}^{4}$ It shows colossal magnetoresistance ${ }^{5}$ and, if appropriately doped, a metal to insulator transition with a resistance change that exceeds 13 orders of magnitude. ${ }^{6,7}$ This renders EuO a very attractive material for basic and device oriented research.

$\mathrm{EuO}$ has been studied extensively as a single crystal $^{5-9}$ and as a thin film, ${ }^{1,3,4,10-14}$ but as a commensurate, epitaxially grown thin film, there is much to be understood about its behavior and films with high structural quality have not, until now, been fabricated. In this letter, we demonstrate the commensurate, epitaxial growth of EuO by molecular-beam epitaxy (MBE) in an adsorption-controlled regime and discuss its crystalline quality, the onset of film relaxation of strained $\mathrm{EuO}$, and finally its magnetic properties.

Although the growth of epitaxial EuO films dates back over 30 years, ${ }^{15}$ its formation is still a difficult balancing act between the europium and oxygen fluxes to avoid the formation of europium metal on the oxygen-poor side and $\mathrm{Eu}_{3} \mathrm{O}_{4}$ or $\mathrm{Eu}_{2} \mathrm{O}_{3}$ on the oxygen-rich side. The traditional approach to the growth of stoichiometric $\mathrm{EuO}$ is to try to precisely match the europium and oxygen fluxes. To avoid flux matching altogether, the use of an adsorption-controlled growth regime has been suggested ${ }^{16}$ and strong indications of its existence have been reported. ${ }^{3}$ Here we prove the existence of an adsorption-controlled growth regime for $\mathrm{EuO}$. By heating the substrate to temperatures high enough to evaporate unoxidized europium metal, the deposition of $\mathrm{EuO}$ can be controlled solely by oxygen flux. This way, all available oxygen reacts to form $\mathrm{EuO}$ and the excess europium evaporates leaving pure stoichiometric $\mathrm{EuO}$.

The films were grown in a Veeco 930 MBE system (described elsewhere ${ }^{17}$ ) with a base pressure of less than

\footnotetext{
a) Electronic mail: rossulbricht@gmail.com.

${ }^{b)}$ Electronic mail: schlom@cornell.edu.
}

$2 \times 10^{-9}$ Torr. We used single crystalline $\mathrm{YAlO}_{3}$ substrates oriented within $\pm 0.5^{\circ}$ of (110). (110) $\mathrm{YAlO}_{3}$ has a rectilinear surface net with in-plane lattice constants of $7.431 \AA$ along

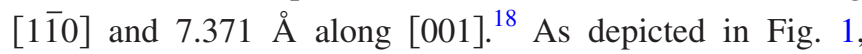
(001) $\mathrm{EuO}$ grows with its unit cell rotated in-plane by $45^{\circ}$ with respect to the (110) $\mathrm{YAlO}_{3}$ surface net. This provides a linear lattice mismatch of $2.2 \%$ for $[110] \mathrm{EuO} \|[1 \overline{1} 0] \mathrm{YAlO}_{3}$ and $1.4 \%$ for $[1 \overline{1} 0] \mathrm{EuO} \|[001] \mathrm{YAlO}_{3}{ }^{1}{ }^{1}$

EuO films were deposited with $\mathrm{O}_{2}$ partial pressures $\left(P_{\mathrm{O}_{2}}\right)$ ranging from $2.5 \times 10^{-10}$ to $1 \times 10^{-8}$ Torr above the background pressure. The substrate temperature $\left(T_{\text {sub }}\right)$ was approximately $590{ }^{\circ} \mathrm{C}$ for all growths, significantly higher than the $T_{\text {sub }} \sim 300{ }^{\circ} \mathrm{C}$ typically used for the epitaxial growth of EuO. ${ }^{111,15,19}$ By choosing a substrate temperature comparable to the temperature of the europium source supplying the europium flux, excess europium metal is readily desorbed from the growing film. The incident europium flux was measured using a quartz crystal microbalance and was adjusted to $1.1 \times 10^{14} \mathrm{Eu}$ atoms $/ \mathrm{cm}^{2} \mathrm{~s}$. A main shutter directly in front of the substrate was opened to initiate growth only after a steady-state oxygen background partial pressure was achieved with the europium source shutter open. The growths were ended $30 \mathrm{~min}$ later by simultaneously closing

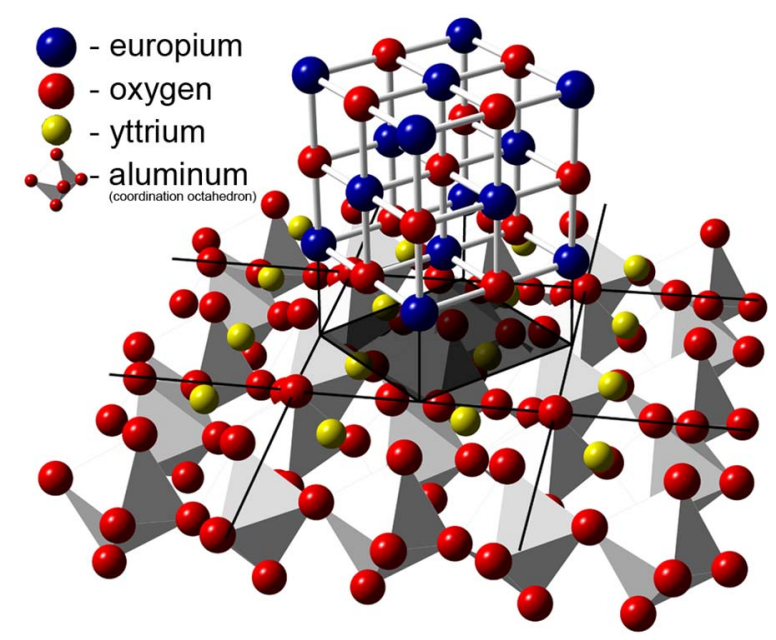

FIG. 1. (Color online) Crystal structure of (001) EuO epitaxially grown on (110) $\mathrm{YAlO}_{3}$ depicting the $45^{\circ}$ rotation of the EuO unit cell (above) with respect to the pseudocubic $\mathrm{YAlO}_{3}$ lattice (below). 

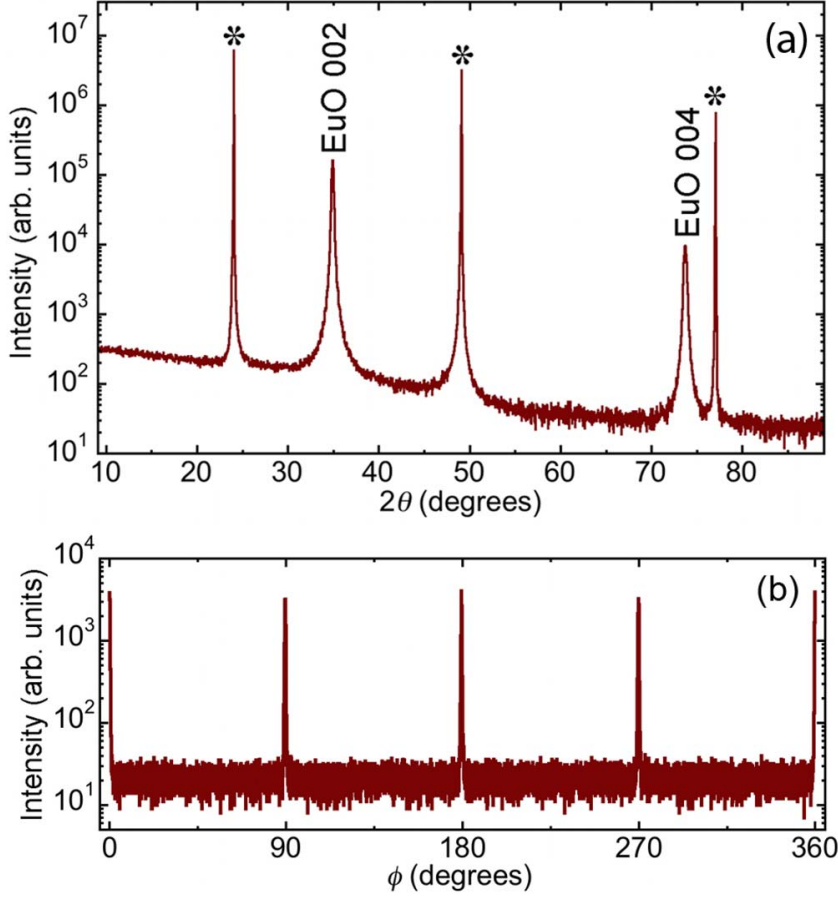

FIG. 2. (Color online) XRD scans from a $356 \pm 4 \AA$ thick EuO film grown on (110) $\mathrm{YAlO}_{3}$ at approximately $590{ }^{\circ} \mathrm{C}$ and $1 \times 10^{-9}$ Torr $P_{\mathrm{O}_{2}}$. (a) $\theta-2 \theta$ scan. (b) Azimuthal $\phi$ scan of the $111 \mathrm{EuO}$ diffraction peaks at $\chi=54.7^{\circ}$. $\chi=0^{\circ}$ aligns the diffraction vector to be perpendicular to the plane of the substrate. $\phi=0^{\circ}$ corresponds to the in-plane component of the diffraction vector aligned parallel to the [001] direction of the (110) $\mathrm{YAlO}_{3}$ substrate. Together, these XRD scans show that the EuO film is epitaxial, commensurate, and oriented with $[001] \mathrm{EuO} \|[110] \mathrm{YAlO}_{3}$ and $[110] \mathrm{EuO} \|[001] \mathrm{YAlO}_{3}$.

the europium source shutter and piezoelectric oxygen valve. After allowing the substrate to cool in ultrahigh vacuum, a $10 \mathrm{~nm}$ thick capping layer of amorphous silicon was deposited at a rate of $1 \times 10^{13} \mathrm{Si}$ atoms $/ \mathrm{cm}^{2} \mathrm{~s}$ to protect the $\mathrm{EuO}$ film from oxidation upon exposure to air. ${ }^{1}$ The crystal growth was monitored in situ using reflection high-energy electron diffraction (RHEED) and ex situ using x-ray diffraction (XRD).

Figure 2 shows XRD data from a $356 \pm 4 \AA$ thick (001)oriented $\mathrm{EuO}$ film grown on (110) $\mathrm{YAlO}_{3}$ at approximately $590{ }^{\circ} \mathrm{C}$ and $P_{\mathrm{O}_{2}}=1 \times 10^{-9}$ Torr. The $\theta-2 \theta$ XRD pattern [Fig. 2(a)] shows that the film is single phase. The azimuthal $\phi$ scan of the $111 \mathrm{EuO}$ peaks [Fig. 2(b)] exhibits four peaks, corroborating the in-plane orientation relationship shown in Fig. 1 and demonstrating that the film is untwined. The inplane lattice spacings of this $\mathrm{EuO}$ film were determined from the $2 \theta$ positions of these $111 \mathrm{EuO}$ peaks in combination with that of the 002 out-of plane EuO peak. Due to the distortion of the $\mathrm{EuO}$ film by the rectilinear surface net of the (110) $\mathrm{YAlO}_{3}$ substrate (with dimensions $7.431 \AA$ along [1] 0 ] $\mathrm{YAlO}_{3}$ and $7.371 \AA$ along [001] $\mathrm{YAlO}_{3}$, which were confirmed by XRD on these substrates), the [110] and [1]0] in-plane $\mathrm{EuO}$ vectors remain perpendicular but are no longer of equal length. The measured spacings of the $\mathrm{EuO}$ surface net were $7.43 \pm 0.07 \AA$ along $[1 \overline{1} 0] \mathrm{EuO}$ and $7.37 \pm 0.07 \AA$ along [110] EuO. The out-of-plane lattice spacing at room temperature was $5.05 \pm 0.08 \AA$, which is in agreement with the value calculated using the in-plane strain and the elastic compliance coefficients for EuO at $77 \mathrm{~K}^{20}$ Together, these $\mathrm{XRD}$ results show that this EuO film is epitaxial, com-

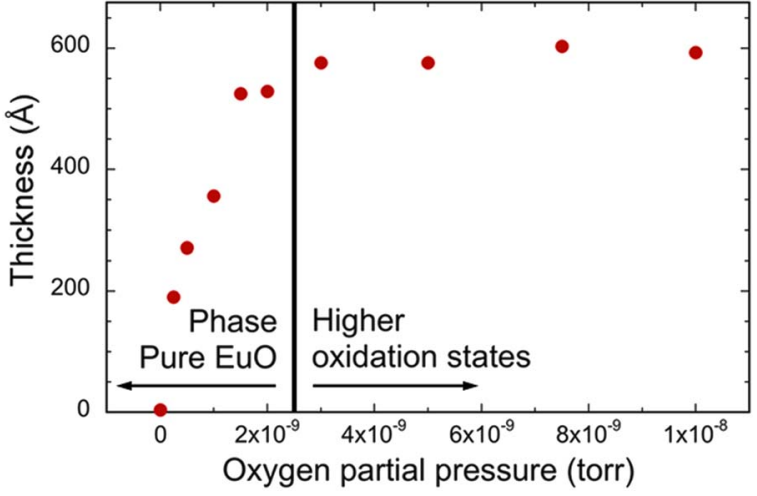

FIG. 3. (Color online) Each film was grown at approximately $590{ }^{\circ} \mathrm{C}$ for 30 minutes in the absorption-controlled regime with a europium flux of 1.1 $\times 10^{14} \mathrm{Eu}$ atoms $/ \mathrm{cm}^{2} \mathrm{~s}$. Below roughly $2.5 \times 10^{-9}$ Torr, the EuO films remain phase pure and additional oxygen reacts with excess europium, increasing the growth rate. At roughly $2.5 \times 10^{-9}$ Torr, the europium and oxygen fluxes are matched and increasing $P_{\mathrm{O}_{2}}$ results in the formation of impurity phases (first $\mathrm{Eu}_{3} \mathrm{O}_{4}$ and then $\mathrm{Eu}_{2} \mathrm{O}_{3}$ ) containing higher oxidation states of europium.

mensurate, and oriented with (001) $\mathrm{EuO} \|(110) \mathrm{YAlO}_{3}$ and $[110] \mathrm{EuO} \|[001] \mathrm{YAlO}_{3}$.

If the film is growing in an adsorption-controlled regime, all incident, unreacted europium desorbs from the film surface. RHEED and XRD were used to show that in the absence of oxygen at $T_{\text {sub }} \sim 590{ }^{\circ} \mathrm{C}$ the incident europium desorbs from the substrate. RHEED imaging of a bare $\mathrm{YAlO}_{3}$ substrate at growth temperature appeared identical before and after exposing it to a europium flux in the absence of oxygen. The sample was capped with silicon and the absence of a europium metal film was confirmed by XRD.

EuO films were grown in differing background partial pressures of oxygen and their europium content was determined using Rutherford backscattering spectrometry (RBS). Using the density of crystalline EuO, this RBS atomic coverage was converted to film thickness. As shown in Fig. 3, as the $P_{\mathrm{O}_{2}}$ used during growth increases, so does the film thickness, indicating a faster growth rate. The observed dependence of film thickness on the oxygen partial pressure (oxygen flux) is a hallmark of adsorption-controlled growth. For films grown with $P_{\mathrm{O}_{2}}$ higher than $2 \times 10^{-9}$ Torr, where the oxygen flux becomes greater than the europium flux, the growth rate saturates, and higher oxidation states (first $\mathrm{Eu}_{3} \mathrm{O}_{4}$ and then $\mathrm{Eu}_{2} \mathrm{O}_{3}$ ) begin to form.

Thin, epitaxial $\mathrm{EuO}$ films are strained in tension due to the mismatch between the substrate and film, while thicker films begin to relax. The film relaxation is accompanied by dislocation and defect formation that results in broadening of the films' rocking curves. To establish the critical thickness where film relaxation begins to occur, single phase $\mathrm{EuO}$ films were grown in the adsorption-controlled regime $\left(T_{\text {sub }}\right.$ $=590{ }^{\circ} \mathrm{C}$ and $P_{\mathrm{O}_{2}}=1 \times 10^{-9}$ Torr) and changes in their rocking curves versus their thicknesses were noted.

Rocking curves from the $002 \mathrm{EuO}$ peaks of films with different thicknesses and the 220 peak of a $\mathrm{YAlO}_{3}$ substrate are shown in Fig. 4(a). Rocking curves were obtained with $\phi$ aligned such that the in-plane component of the diffraction vector is parallel to one of the four $\langle 100\rangle$-type $\mathrm{EuO}$ in-plane directions that are equally strained. Films grown thinner than the thickness at which relaxation begins under these growth conditions display sharp rocking curves with full width at 

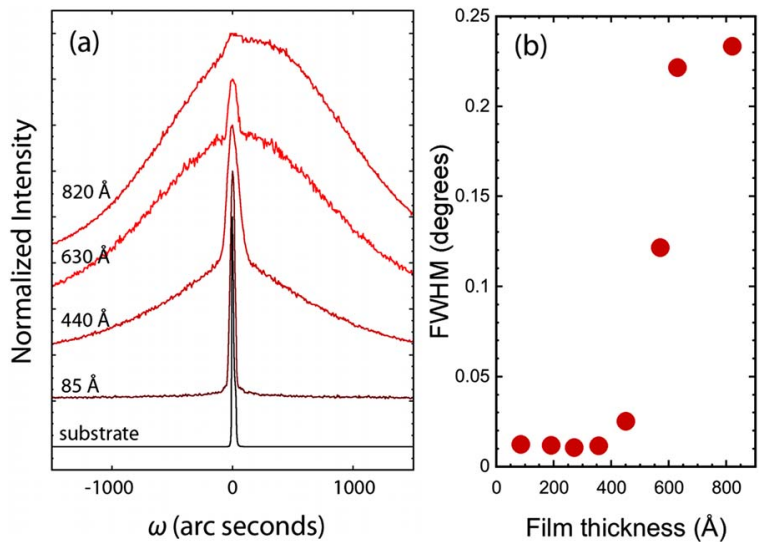

FIG. 4. (Color online) (a) Rocking curves from the $002 \mathrm{EuO}$ peaks of films with different thicknesses and the 220 peak of a $\mathrm{YAlO}_{3}$ substrate. Two superimposed peak shapes near $440 \AA$ indicate film relaxation through misfit dislocations. The peak intensities are normalized and shifted relative to one another for clarity. (b) FWHM of the $002 \mathrm{EuO}$ film peak as a function of the film thickness. Narrow FWHMs comparable to that of the 220 peak of the $\mathrm{YAlO}_{3}$ substrates are maintained for $\mathrm{EuO}$ film thicknesses below $\sim 380 \AA$ A. The FWHM begins to broaden beyond this thickness.

half maximum (FWHM) as narrow as 34 arc sec, which is comparable to those of the substrates (12-34 arc sec). Partially relaxed films show the same sharp peaks on top of a broad background peak originating from the relaxed portion of the film. As the films grow beyond the onset of relaxation, the intensity of the broad background peak increases as defects are formed and the intensity of the sharp central peak decreases as less and less of the film is commensurately strained. The broad background peak also shifts to higher values of omega indicating an expansion of the out-of-plane lattice constant during relaxation. As discussed previously, the $\mathrm{EuO}$ in-plane lattice spacings of a $356 \pm 4 \AA$ thick film closely matched those of the (110) $\mathrm{YAlO}_{3}$. In contrast, the in-plane spacings of an $820 \pm 8 \AA$ thick EuO film were determined to be $7.28 \pm 0.03 \AA$ along [1히 $\mathrm{EuO}$ and $7.28 \pm 0.03 \AA$ along [110] EuO. The out-of-plane lattice spacing was $5.14 \pm 0.013$. Figure $4(\mathrm{~b})$ shows the rocking curve FWHM of the $002 \mathrm{EuO}$ film peak as a function of film thickness. For $d$ less than $356 \pm 4 \AA$, the films maintain a narrow rocking curve. For $d$ greater than $440 \pm 4 \AA$, the rocking curve FWHM begins to broaden in a manner consistent with other epitaxial semiconductor and oxide films undergoing relaxation. ${ }^{21-23}$ From $d=820 \pm 8 \AA$ and beyond, no commensurate central peak is observed in the rocking curves. The onset of film relaxation agrees well with the onset of broadening of the FWHM of the rocking curves.

The magnetic properties of a coherently strained $\mathrm{EuO}$ film were measured using a superconducting quantum interference device. As seen in Fig. 5, the ferromagnetism sets in at $69 \mathrm{~K}$ when cooled without an external magnetic field and grows to $6.96 \pm 0.07 \mu_{B}$ per $\mathrm{Eu}$ atom under a saturating external magnetic field at $5 \mathrm{~K}$. This is consistent with single crystalline EuO (Ref. 2) and is the highest reported saturated magnetization for a thin film of EuO.

These films exhibit rocking curve FWHMs approaching those of the $\mathrm{YAlO}_{3}$ substrates on which they are grown. High quality films such as these minimize the extrinsic effects arising from defects and will allow the fundamental

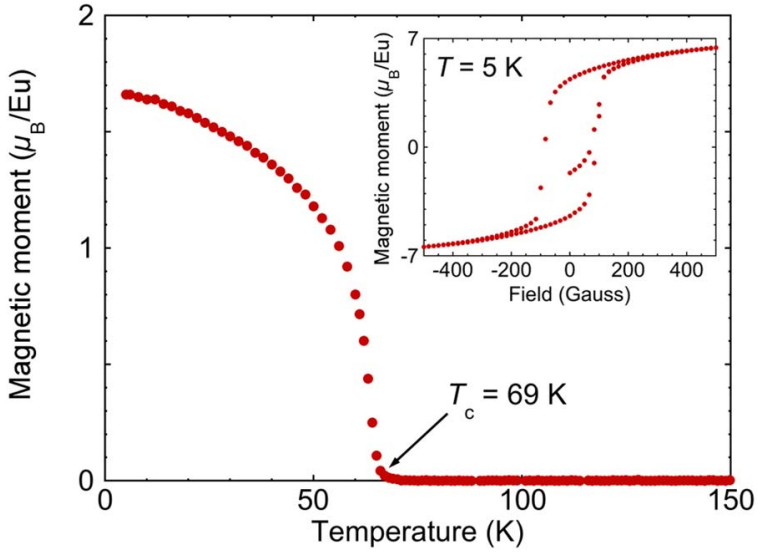

FIG. 5. (Color online) Zero-field temperature dependence of the magnetic moment of a $271 \pm 3 \AA$ thick, commensurately strained EuO film grown on (110) $\mathrm{YAlO}_{3}$. The onset of ferromagnetism occurs at $69 \mathrm{~K}$. At $5 \mathrm{~K}$, the magnetic hysteresis (inset) shows ferromagnetic behavior with a saturation magnetization of $6.96 \pm 0.07 \mu \mathrm{B}$ per europium atom, $99.5 \%$ of its theoretical maximum.

properties of EuO (e.g., the dependence of $T_{c}$ on $\operatorname{strain}^{24}$ ) to be established.

The authors gratefully acknowledge informative discussions with George Sawatzky. This work was supported by the Office of Naval Research through Grant Nos. N0001403-1-0721 and N00014-04-1-0426 monitored by Colin Wood and by the NSF through Grant Nos. DMR-0213623 and DMR-0507146. The work at the University of Augsburg was supported by the EU (Nanoxide) and the DFG (SFB 484).

${ }^{1}$ A. Schmehl, V. Vaithyananthan, A. Herrnberger, S. Thiel, C. Richter, M. Liberati, T. Heeg, M. Röckerath, L. F. Kourkoutis, S. Mühlbauer, P. Böni, D. A. Muller, Y. Barash, J. Schubert, Y. Idzerda, J. Mannhart, and D. G. Schlom, Nat. Mater. 6, 882 (2007).

${ }^{2}$ B. T. Matthias, R. M. Bozorth, and J. H. Van Vleck, Phys. Rev. Lett. 7, 160 (1961).

${ }^{3}$ P. G. Steeneken, Ph.D. thesis, Rijksuniversiteit Groningen, 2002.

${ }^{4}$ K. Y. Ahn and M. W. Shafer, J. Appl. Phys. 41, 1260 (1970).

${ }^{5}$ M. R. Oliver, J. O. Dimmock, A. L. McWhorter, and T. B. Reed, Phys. Rev. B 5, 1078 (1972).

${ }^{6}$ G. Petrich, S. von Molnár, and T. Penney, Phys. Rev. Lett. 26, 885 (1971). ${ }^{7}$ M. W. Shafer, J. B. Torrance, and T. Penney, J. Phys. Chem. Solids 33, 2251 (1972).

${ }^{8}$ K. Sattler and H. C. Siegmann, Phys. Rev. Lett. 29, 1565 (1972).

${ }^{9}$ H. A. Mook, Phys. Rev. Lett. 46, 508 (1981).

${ }^{10}$ K. Y. Ahn and J. C. Suits, IEEE Trans. Magn. 4, 408 (1968).

${ }^{11}$ C. Llinares, L. Gouskov, C. Duchemin, and G. Bordure, J. Phys. Chem. Solids 36, 567 (1975).

${ }^{12}$ N. Iwata, G. Pindoria, T. Morishita, and K. Kohn, J. Phys. Soc. Jpn. 69, 230 (2000).

${ }^{13}$ P. G. Steeneken, L. H. Tjeng, I. Elfimov, G. A. Sawatzky, G. Ghiringhelli, N. B. Brookes, and D. J. Huang, Phys. Rev. Lett. 88, 047201 (2002).

${ }^{14}$ J. Lettieri, V. Vaithyanathan, S. K. Eah, J. Stephens, V. Sih, D. D. Awschalom, J. Levy, and D. G. Schlom, Appl. Phys. Lett. 83, 975 (2003).

${ }^{15}$ K. Lee and J. C. Suits, J. Appl. Phys. 41, 954 (1970).

${ }^{16}$ H. Ott, S. J. Heise, R. Sutarto, Z. Hu, C. F. Chang, H. H. Hsieh, H. J. Lin, C. T. Chen, and L. H. Tjeng, Phys. Rev. B 73, 094407 (2006).

${ }^{17}$ C. D. Theis and D. G. Schlom, J. Cryst. Growth 174, 473 (1997).

${ }^{18}$ G. M. Roesler Jr., Y. U. Idzerda, P. R. Broussard, and M. S. Osofsky, J. Appl. Phys. 75, 6679 (1994).

${ }^{19}$ R. Diehl and G. Brandt, Mater. Res. Bull. 10, 85 (1975).

${ }^{20}$ Y. Shapira and T. B. Reed, AIP Conf. Proc. 5, 837 (1971).

${ }^{21}$ P. F. Miceli and C. J. Palmstrøm, Phys. Rev. B 51, 5506 (1995).

${ }^{22}$ C. Kim, T. Spila, I. K. Robinson, and J. E. Greene, J. Appl. Phys. 83, 7608 (1998).

${ }^{23}$ A. R. Kortan, M. Hong, J. Kwo, J. P. Mannaerts, and N. Kopylov, Phys. Rev. B 60, 10913 (1999).

${ }^{24}$ N. J. C. Ingle and I. S. Elfimov, Phys. Rev. B 77, 121202 (2008). 